\title{
The Deubiquitin Enzyme USP28 Maintains the Viability of Triple Negative Breast Cancer Cells through Stabilizing the RecQ Family Helicases
}

\section{Jin Peng}

First Hospital of Zhejiang Province: Zhejiang University School of Medicine First Affiliated Hospital Jiewei Wang

First Hospital of Zhejiang Province: Zhejiang University School of Medicine First Affiliated Hospital

\section{Yiping Dong}

Xi'an Jiaotong University Medical College First Affiliated Hospital

\section{Huailu Ma}

Zhejiang University

\section{Lingzhi Wu}

First Hospital of Zhejiang Province: Zhejiang University School of Medicine First Affiliated Hospital

\section{Xinghua Zhen}

First Hospital of Zhejiang Province: Zhejiang University School of Medicine First Affiliated Hospital

\section{Lichun Tang}

State Key Laboratory of Proteomics: Beijing Proteome Research Center

Jianping Jin

Zhejiang University Life Science Institute

\section{Suxia Han}

Xi'an Jiaotong University Medical College First Affiliated Hospital

Pumin Zhang ( $\sim$ pzhangbcm@zju.edu.cn)

Zhejiang University School of Medicine First Affiliated Hospital https://orcid.org/0000-0003-4063-3008

\section{Research article}

Keywords: USP28, Triple-negative breast cancer, Deubiquitin enzyme, RecQ family helicases, RECQL5

Posted Date: July 7th, 2021

DOI: https://doi.org/10.21203/rs.3.rs-645209/v1

License: (c) (1) This work is licensed under a Creative Commons Attribution 4.0 International License.

Read Full License 


\title{
The Deubiquitin Enzyme USP28 Maintains the Viability of Triple Negative Breast Cancer Cells through Stabilizing the RecQ Family Helicases
}

\author{
Jin Peng a, b, \#, Jiewei Wang ${ }^{\mathrm{c}, \# \text {, Yiping Dong }}{ }^{\mathrm{f}}$, Huailu Ma ${ }^{\mathrm{b}}$, Lingzhi Wu ${ }^{\mathrm{a}, \mathrm{b}}$, Xinghua \\ Zhen $^{\text {a, b }}$, Lichun Tang e , Jianping Jin ${ }^{\mathrm{g}}$, Suxia Han ${ }^{\mathrm{f}}$, Pumin Zhang a, b, d, e *
}

${ }^{a}$ Zhejiang Provincial Key Laboratory of Pancreatic Disease, The First Affiliated Hospital of Zhejiang University, Hangzhou, Zhejiang 310003, China.

${ }^{\mathrm{b}}$ Institute of Translational Medicine, Zhejiang University Medical School, Hangzhou, Zhejiang 310058, China.

${ }^{c}$ Department of Gastroenterology, The First Affiliated Hospital of Zhejiang University, Hangzhou, Zhejiang 310003, China.

${ }^{d}$ Cancer Center, Zhejiang University, Hangzhou, Zhejiang 310058 China

${ }^{\mathrm{e}}$ State Key Laboratory of Proteomics, National Center for Protein Sciences (Beijing), Beijing Proteome Research Center, Beijing Institute of Lifeomics, Beijing 102206, China

${ }_{\mathrm{f}}^{\mathrm{f}}$ Department of Oncology, The First Affiliated Hospital, Xi'an Jiaotong University Medical College, Xi'an 710061, China.

${ }^{\mathrm{g}}$ Life Sciences Institute, Zhejiang University, Hanzhou, Zhejiang 310058, China

\#: These authors contributed equally to the work.

Running title: USP28 in triple negative breast cancer

Address Correspondence to: Pumin Zhang, Ph.D.

Zhejiang Provincial Key Laboratory of Pancreatic Disease The Frist Affiliated Hospital of Zhejiang University

Hangzhou, Zhejiang 310003

China

E-mail: pzhangbcm@zju.edu.cn 


\begin{abstract}
Background: Triple-negative breast cancer lacks significant expression of estrogen receptor, progesterone receptor, and human epidermal growth factor receptor 2 . It is the more aggressive and malignant kind of breast cancers and is currently without any effective targeted therapies.
\end{abstract}

Methods: We have screened for genes in the ubiquitin-proteasome system that are essential for the proliferation and survival of TNBC cells via CRISPR/Cas9-mediated gene editing. Growth of TNBC cells were assayed using cell and tumor xenograft models to validate the vital role of USP28. We employed cell biology and biochemical methods to uncover the mechanisms underlying the requirement of USP28 for the proliferation of TNBC cells.

Results: USP28, a deubiquitin enzyme, is an essential gene for TNBC cells in vitro and in vivo. Compromising the function of USP28 causes TNBC cells to arrest in $\mathrm{S} / \mathrm{G} 2$ phases with DNA damage checkpoint activation. We show that RecQ family helicases are regulated by USP28, which is more important in TNBC cells than in other breast cancer cells. We further showed that a small molecule inhibitor of USP28 displayed antitumor activities against xenografts derived from TNBC cells.

Conclusion: Our data establish a critical role played by USP28 in supporting the proliferation and viability of triple negative breast cancer cells through stabilizing RecQ family helicases and support USP28 as a therapeutic target for TNBC.

Keywords: USP28, Triple-negative breast cancer, Deubiquitin enzyme, RecQ family helicases, RECQL5 


\section{Introduction}

Triple-negative breast cancer (TNBC) lacks significant expression of estrogen receptor (ER), progesterone receptor (PR), and human epidermal growth factor receptor 2 (HER2) [1]. Although it is only 10-20\% of all breast cancers, TNBC is more aggressive and poorer in prognosis than other breast malignancies. Gene expression profiling of TNBC showed enrichment of genes involved in DNA damage checkpoint response [2, 3], which is consistent with the finding of higher levels of endogenous DNA damage in TNBC cells in vitro and in vivo $[4,5]$. Moreover, about 20\% TNBC patients carry mutation in BRCA1/2 [6, 7]. While hormonal and targeted therapies are available for ER positive and HER2 positive breast cancers, triple negative breast cancer currently lacks targeted therapies (except BRCA1/2 mutated ones) and relies on chemotherapy [8].

USP28 is a ubiquitin-specific cysteine protease (USP) and has been shown to play an important role in maintaining the stability of a large number of proteins including cMYC [9], LSD1 [10], HIF1alpha [11], Notch1 [12], 53BP1 [13], and CLASPIN [14]. Almost all these substrates, especially c-MYC, have been demonstrated to contribute significantly to the initiation and progression of various types of cancers. Consistent with that, Usp28 was shown to help the tumorigenesis of colorectoral cancer in mice [12]. More recently, USP28 was shown to be required by squamous cell carcinoma (SCC) due to its function in maintaining stability of Np63 [15].

Here we report that USP28 is required for the viability of triple negative breast cancer cells and this requirement is not only associated with its function in maintaining 
the stability of c-MYC protein but more importantly with its role in protecting RECQL5 and other members of the RecQ family helicases from proteasome-mediated degradation. These helicases are required for TNBC cells to deal with the elevated levels of endogenous DNA damage resulted from replication stress [5, 16]. As a result, compromising the function of USP28 causes TNBC cells to arrest in S/G2 phases with DNA damage checkpoint activation. We further showed that a small molecule inhibitor of USP28 displayed anti-tumor activity against xenografts derived from TNBC cells. Our results point USP28 as a therapeutic target for triple negative breast cancer.

\section{Materials and Methods}

Cell culture

MDA-MB-231, MDA-MB-468, HCC1806, HCC38, BT549, SUM159, T47D and IMR90 cell lines were purchased from American Type Culture Collection (ATCC). HCC1937, MCF7 and 293T cells were obtained from The Cell Bank of Type Culture Collection of Chinese Academy of Sciences (Beijing, China). The above cells were cultured in DMEM, RPMI-1640 or F12 Medium with 10\% FBS in a humidified atmosphere containing $5 \% \mathrm{CO}_{2}$ at $37^{\circ} \mathrm{C}$. Dulbecco's modified Eagle's medium (DMEM), RPMI-1640 Medium, F12 medium and fetal bovine serum (FBS) were purchased from Gibco (Grand Island, New York, USA).

Reagents

The antibodies used in this study were as follows: USP28 (17707-1-AP, 1:1000 WB, Proteintech, Wuhan, China); RECQL1 (A300-450A, 1:1000 WB, Bethyl Lab, 
Montgomery, TX, USA); WRN ( 4666S,1:1000 WB ,Cell Signaling, Beverly, MA, USA); BLM (A300-110A ,1:1000 WB, Bethyl Lab); RECQL4 (17008-1-AP ,1:1000 WB, Proteintech); RECQL5 (A302-520A, 1:2,000 WB, Bethyl Lab); Flag (F3165, 1:2000, Sigma); Ub (SC-8017, 1:200 WB, Santa Cruz Biotechnology, CA); $\gamma \mathrm{H} 2 \mathrm{AX}$ (05636, 1:500 IF, Millipore, Billerica, MA, USA); Claspin (2800S, 1:1000 WB, Cell Signaling); c-MYC (SC-40, 1:200 WB, Santa Cruz); Phospho-Chk1-Ser345 (2348, 1:1000 WB, Cell Signaling); CHK1 (ab32531, 1:1000 WB, Abcam, Cambridge, UK); GAPDH (60004-1-1g, 1:5000 WB, Proteintech, Wuhan, China); Tubulin (66240-1-1g, 1:5000 WB, Proteintech); Actin (66009-1-1g, 1:3000 WB, Proteintech). Secondary antibodies conjugated to horseradish peroxidase for western blot and Secondary antibodies anti-mouse, -goat or -rabbit Alexa fluor 488 or 594 for immunofluorescence staining were purchased from Jackson ImmunoResearch Laboratories (West Grove, Pennsylvania, USA). siRNAs were synthesized by GenePharma (Suzhou, China). Chemicals 5-Fluorouracil (5-FU) and camptothecin (CPT) were obtained from Selleck (Houston, TX, USA).

\section{Plasmids and lentiviruses}

Plasmids used in the work were generated through standard cloning methods. shRNAs were constructed in pLKO.1 with following sequences:

\begin{tabular}{|l|l|}
\hline Gene & Sequence \\
\hline negative control & 5'-TTCTCCGAACGTGTCACGT-3' \\
\hline shUSP28-1 & 5'-GCACAGAAGTTCGTTGTCATA-3' \\
\hline shUSP28-2 & 5'-GACTGAAGATCATCCATTAAT-3' \\
\hline
\end{tabular}




\begin{tabular}{|l|l|}
\hline shRECQL1-1 & 5'-GCACATGCTATTACTATGCAA-3' \\
\hline shRECQL1-2 & 5'-GCCCTCAAACACTGAAGATTT-3' \\
\hline shWRN-1 & 5'-GCTGGCAATTACCAGAACAAT-3' \\
\hline shWRN-2 & 5'-GAGGGTTTCTATCTTACTAAA-3' \\
\hline shBLM-1 & 5'-GACGCTAGACAGATAAGTTTA-3' \\
\hline ShBLM-2 & 5'-TCACAAGGAATGAGAAATA-3' \\
\hline shRECQL4-1 & 5'-CTAGGAAGAGCCTCATCTAAG-3' \\
\hline shRECQL4-2 & 5'-CGGCTCAACATGAAGCAGAAA-3' \\
\hline
\end{tabular}

The USP28 cDNA (wild-type or C171A mutant) and RECQL5 cDNA were

cloned into lentiviral vector pHAGE. The $c-M Y C$ cDNA (wild-type or T58A nutant) were cloned into pCMV.

USP28 sgRNA was cloned into the lentiviral vector pLKO-U6-sgRNA with the following sequence: 5'-ACCCCAATCCCAATGACTGG-3'.

Lentiviruses-carrying overexpression, knockdown or knockout elements were produced in the lab and used to infect the above cell lines with MOI (multiplicity of infection) $>1$. The infected cells were selected with puromycin treatment $(4 \mu \mathrm{g} / \mathrm{ml}$ for 2 days).

Loss of function screening based on CRISPR-Cas 9

For Ubiquitin proteasome system (UPS) -wide genetic screen, the human sgRNA library targeting UPS (containing 2197 sgRNAs targeting 1098 UPS related genes and 1 negative control) was constructed into pLKO-U6-sgRNA. 
6 million of HCC1937 cells were infected with UPS sgRNA lentiviral pool at a MOI of 0.1-0.3 to achieve an average coverage of 300-fold of the library after puromycine selection. 3 million sgRNA expressing cells were infected with adenovirus expressing GFP or Cas9(>30 million copies). After 10 population doublings of the cells, a final pool of 3 million cells was harvested. Genomic DNA was extracted using TIANamp Genomic DNA Kit (Tiangen). sgRNA inserts were PCR amplified using KOD FX (TOYOBO) from 2 million genome equivalents of DNA to ensure an average coverage of 1000x of the library. Primer sequences used for amplification of sgRNA inserts are:

\begin{tabular}{|l|l|}
\hline Forward & 5'-GCATATACGATACAAGGCTG-3' \\
\hline Reverse & 5'-AAGTTGATAACGGACTAGCC-3' \\
\hline
\end{tabular}

The PCR products were purified and subjected to deep sequencing. sgRNA abundance in both GFP and Cas9 treated groups were calculated and analyzed.

\section{Assays for cell proliferation}

For MTS assay, after lentiviral infection and selection, the cells were trypsinized and re-seeded in 96-well plates at a density of 3,000 cells/well and cultured for the indicated time. At the end of incubation, the number of viable cells was analyzed using a colorimetric assay (MTS, Promega, Madison, WI, USA). Briefly, $20 \mu \mathrm{l}$ MTS was added to $100 \mu \mathrm{l}$ fresh complete culture medium in each well, and the cells were incubated for 2 hrs before the absorbance of the formazan product at $490 \mathrm{~nm}$ was measured.

To detect the effects of replication inhibitors on non-TNBC cell viability, T47D cells were seeded at 5,000 cells/well in 96-well plates and then treated with various 
concentrations of CPT $(0,5,10,15,20 \mathrm{nM})$ or 5 -FU $(0,25,50,100,200 \mu \mathrm{g} / \mathrm{ml})$ for 48hrs. Following the treatment, MTS assays were performed.

\section{Fluorescence activated cell sorting}

The cells were trypsinized and washed once with cold PBS. For cell cycle analysis, the cells were fixed in 70\% ice-cold EtOH, spun down, washed with cold PBS, and incubated in PBS containing propidium iodide (PI, $50 \mu \mathrm{g} / \mathrm{ml}$ ) and RNase A (50 $\mu \mathrm{g} / \mathrm{ml}$ ) for $30 \mathrm{~min}$ at room temperature. The PI-stained single cell suspension was analyzed on a BD LSRFortessa SORP Flow Cytometer (BD Biosciences). ModFit LT software (Verity Software House, Topsham, ME, USA) was used to analyze the DNA pattern and cell cycle stages.

\section{Western blotting analysis}

The cells were lysed with RIPA buffer (Applygen Technologies Inc., Beijing, China) supplemented with a protease inhibitor cocktail (Roche Diagnostics, Mannheim, Germany). Western blots were processed according to standard procedures and analyzed with Chemiluminescent imaging system LAS 500 (GE Healthcare).

\section{Immunoprecipitation}

HEK293T cells cultured in $10 \mathrm{~cm}$ dish were transfected with $5 \mu \mathrm{g}$ Flag-RECQL5 using PEI (Polysciences Inc, Warrington, PA, USA). After 48hrs, harvest the cells and lysate them in NETN lysis buffer. The Flag M2 beads (Sigma) were washed with NETN lysis buffer. The protein A/G beads were washed and then mixed with Rabbit IgG as 
negative control or USP28 antibodies. About $2 \mu \mathrm{g}$ total protein from the cell lysates were incubated with the beads prepared above for 3 hours at $4^{\circ} \mathrm{C}$. The bound proteins were denatured by adding $50 \mu \mathrm{l}$ SDS loading buffer and boiling for 5 minutes, and then analyzed by immunoblotting.

\section{In vivo ubiquitination assay}

HEK293T cells were transfected with Flag-RECQL5 and other indicated plasmids for $48 \mathrm{hrs}$ followed by the treatment with $10 \mu \mathrm{M}$ MG132 for $8 \mathrm{hrs}$. Lyse the cells in denaturing buffer containing $1 \%$ SDS and $1 \%$ sodium deoxycholate, vortex vigorously for 15 30 minutes, boil the cell lysates at $100^{\circ} \mathrm{C}$ for 10 minutes and then add $5 \mathrm{x} \sim 9 \mathrm{x}$ volumes of NETN buffer. The cell lysates were incubated with Flag M2 beads overnight at $4^{\circ} \mathrm{C}$ followed by washing and western blotting analysis.

\section{Immunostaining}

The cells after the indicated treatment were plated on coverslips, fixed with $4 \%$ paraformaldehyde for 15 min, permeabilized in PBS containing 0.5\% Triton X-100 for 5 min, and blocked with 5\% BSA in PBS for $1 \mathrm{~h}$ at room temperature, followed by incubation with primary antibodies at $4^{\circ} \mathrm{C}$ overnight. After 3 washes in PBS, the coverslips were incubated with secondary antibodies for $20 \mathrm{~min}$ at $37^{\circ} \mathrm{C}$. All images were taken on a Nikon Ni-E microscope (Nikon Corporation, Tokyo, Japan), with identical exposure times for each sample.

\section{Tumor xenograft}


$2 \times 10^{6}$ HCC1806 cells were infected with lentiviruses expressing USP28 or control shRNAs and used to inoculate BALB/c nude mice the left inguen. The mice are purchased at 6 8 week of age from Beijing Vital River Laboratory Animal Technology Co., Ltd. All animals were kept in an environmentally controlled facility and given free access to water and a standard diet. Three weeks after the inoculation, the tumors were

removed, and the tumor weight were measured. To evaluate the anti-tumor effect of AZ1, HCC1806 cells similarly inoculated and allowed to grow for 10 days when the tumor size had reached $100 \mathrm{~mm}^{3}$. The tumor-bearing mice were then randomized into two groups and dosed daily by oral gavage at $200 \mathrm{mg} / \mathrm{kg}$ of AZ1 or the vehicle. The tumor growth was monitored every three days using vernier caliper. Two weeks after the treatment, the mice were sacrificed. The tumors were excised out and weighed. All animal experiments were performed according to the guidelines approved by the Animal Care and Use Committee of the First Affiliated Hospital of Zhejiang University.

\section{Statistics}

Statistics analyses were performed with GraphPad Prism9.0 and ImageJ. Unpaired two-tailed Student's $t$ test was used to analyse the significance between two groups. $\mathrm{P}<0.05$ was considered as statistical significance $(*, \mathrm{p}<0.05 ; * *, \mathrm{p}<0.01 ; * * *$, $\mathrm{p}<0.001)$. 


\section{Results}

\section{Identification of USP28 as an essential gene in TNBC cell lines}

We performed a CRISPR/Cas9-based genetic screen to identify genes essential for the survival/proliferation of triple negative breast cancer cells. We used the BRCA1 deficient line HCC1937 for the screening. The gRNA library was designed against genes involved in the ubiquitin proteasome system and constructed in a lentiviral vector with 2 gRNAs for each gene. The cells were infected with the library viruses with a MOI of 0.1 0.3 and with more than 300 times coverage of the library. The infected cells were selected for by the expression of puromycin resistance for the viral vector and split into two halves which were infected with adenoviruses carrying Cas9 or GFP expression cassettes, respectively (Fig. S1A). After the adenoviral infection, the cells were cultured for more than 10 population doublings before being harvested for genomic DNA extraction, and PCR amplification and deep sequencing of the gRNAs. The abundance (reads) of each gRNA was normalized against total reads of each population of the cells (Cas9 or GFP) and compared between the two populations. The result is summarized in a volcano plot (Fig. S1B). Among the gRNAs with more than 3-fold negative enrichment in the Cas9 population than the control GFP population, we chose the one gRNA against USP28 for further study. Another gRNA against USP28 in the library was not enriched, probably due to its low efficiency in editing.

To validate the screening results, we expressed USP28 gRNA and Cas9 in HCC1937 cells, the line used in the original screen, and checked the expression levels of USP28. As expected, this sgRNA could block USP28 expression (Fig. S1C), confirming 
its effectiveness in editing USP28. Furthermore, HCC1937 plus another TNBC cell line MDA-MB-231 which is proficient in BRCAl function, and a non-TNBC cell line MCF7, were employed to examine the effects of this sgRNA on their proliferation. As shown in Fig. S1D, the proliferation of both MDA-MB-231 and HCC1937 cells was suppressed after Cas9 expression, but not that of MCF7 cells, indicating that USP28 is specifically required in TNBC cells, and this requirement is independent of the BRCAl status of the cell.

\section{USP28 is required for the proliferation of triple negative breast cancer cells}

To determine if knocking down USP28 expression with shRNA could also achieve the effect of the gene editing, we designed two shRNAs against the 3'-UTR region of USP28 and used them to deplete USP 28 expression in a number of TNBC cell lines. Knocking down the expression of USP28 indeed could block the proliferation of these TNBC cell lines (Fig. 1A and Fig. S2 A-D), but could block neither the proliferation of MCF7 (Fig S2E) nor that of MCF10A cells (Fig. S2F). Importantly, the effect of these two shRNAs on proliferation could be rescued by re-expression of USP 28 (Fig. 1B and C). Further, we synthesized the reported USP28 inhibitor AZ1 [17] and used it to treat TNBC cells. In HCC1806 cells, a 48-hour AZ1 treatment resulted in a dose-dependent suppression of proliferation (Fig. 1D-E), and similar results were obtained with MDA-MB-231 and HCC1937 (Fig. 1F).

To demonstrate that USP28 is required for the proliferation of TNBC cells in vivo, we depleted its expression in HCC1806 cells via the two shRNAs above and inoculated 
the cells in female nude mice. As expected, these cells could not grow as xenografts (Fig. 2A). Further, we used AZ1 to treat mice carrying HCC1806 xenografts. Although AZ1 is not a potent USP28 inhibitor, it did show anti-TNBC tumor activities (Fig. 2B).

These results demonstrate that UPS28 is uniquely required by the triple negative breast cancer cells for proliferation in vitro and in vivo, but not required by ER positive breast cancer cells nor by the normal mammary epithelial cells, although USP28 is expressed at similar levels across these cell lines (Fig. S2G).

\section{The requirement of USP28 in TNBC cells could not be bypassed with MYC expression}

USP28 was shown previously to regulate the stability of c-MYC protein [9]. We therefore set out to determine if it does so in triple negative breast cancer cells as well. As shown in Fig. 3A, depleting USP28 did cause decreases in c-MYC levels in TNBC cells. However, c-MYC levels did not decline in MCF7 cells (Fig. 3A), which is consistent with the finding that USP28 loss of function, either through Cas9-mediated editing (Fig. S1D) or shRNA-mediated depletion (Fig. S2E), had no effect on their proliferation. Thus, it is possible that the depletion of USP28 in triple negative breast cancer cells reduces cMYC to a level insufficient to support proliferation, making USP28 essential in these cells. To determine if that is the case, we first analyzed cell cycle distribution in USP28 depleted cells. Given the function of c-MYC in promoting G1/S transition, it was expected that USP28-depleted TNBC cells would show a G1 arrest. However, we saw an increase in G2/M fraction instead (Fig. 3B), while MCF7 cells did not show any cell cycle disturbance (Fig. 3B). Furthermore, in both MDA-MB-231 and HCC1937 cells, S 
phase fraction also increased upon USP28 depletion (Fig. 3B). These results suggest USP28 regulate more than c-MYC in TNBC cells to help their proliferation.

We next sought to determine if re-express $c-M Y C$ could rescue USP28 depletion. However, we were unable to express wildtype $c-M Y C$ in $U S P 28$-depleted cells, as the wildtype c-MYC protein relies on USP28 for stability. To circumvent that, we expressed $c-M Y C^{T 58 A}$, a mutant form resistant to ubiquitination catalyzed by FBXW7 [18, 19]. $c$ $M Y C^{T 58 A}$ was overexpressed in MDA-MB-231 cells first, and USP28 expression was subsequently depleted via shRNA (Fig. 3C). Cell proliferation assay on these cells demonstrated that restoring $c-M Y C$ expression could not prevent these cells from losing the ability to proliferate due to USP28 depletion (Fig. 3C). However, the exogenously expressed $c-M Y C^{T 58 A}$ did not cause an increase in the rate of proliferation neither (Fig. 3C), which made us wander whether the $c-M Y C^{T 58 A}$ expressed was functional. To rule that out, we expressed the same $c-M Y C^{T 58 A}$ in human normal diploid fibroblasts IMR90. Both wildtype $c-M Y C$ and $c-M Y C^{T 58 A}$ could enhance the proliferation of IMR90 cells, and $c-M Y C^{T 58 A}$ showed a stronger effect than the wildtype (Fig. S4). Thus, the $c-M Y C^{T 58 A}$ expression construct was functional. It is likely that MDA-MB-231 cells express already high enough levels of $c-M Y C$, and thus further increasing its expression levels would not be able to further stimulate proliferation. Taken together, these results indicate that the reduction in c-MYC stability resulted from USP28 depletion is not the reason for triple negative breast cancer cells to cease proliferation.

\section{Increased levels of endogenous DNA damage in USP28-depleted TNBC cells}


The cell cycle profiles of USP28-depleted TNBC cells (Fig. 3B) suggest DNA damage checkpoint activation that stalled the cells in S and G2 phases. Together with our previous report of increased levels of DNA damage in TNBC cells due to elevated levels of replication stress [5], we decided to examine DNA damage levels in USP28-depleted TNBC cells. Staining of $\gamma \mathrm{H} 2 \mathrm{AX}$ showed that indeed the endogenous DNA damage levels increased significantly, from $30 \%$ in the control to more than $60 \%$ of $\gamma \mathrm{H} 2 \mathrm{AX}$ positive cells when USP28 was depleted (Fig. 4A). Consistent with that, the level of phosphorylated CHK1 also increased significantly (Fig. 4B), indicating activation of DNA damage checkpoint. These results provide an explanation for the observed stall in $\mathrm{S}$ and $\mathrm{G} 2$ phases (Fig. 3B).

USP28 was reported to regulate the stability of CLASPIN [14] which is involved in DNA damage response in S phase. We therefore hypothesized that such a regulation is critical in TNBC cells to relieve replication stress and prevent subsequent DNA damages. However, the levels of CLASPIN did not change upon USP28 depletion (Fig. 4C), suggesting other substrates of USP28 are more important in this regard in TNBC cells.

We showed previously that the function of RECQL5 was critical in triple negative breast cancer cells [5] and therefore decided to look into the possibility that USP28 regulates its stability. As shown in Fig. 4D, depletion of USP28 led to a significant decrease in the levels of RECQL5 in TNBC and non-TNBC (T47D) cells. Furthermore, the other family members of $R E C Q L 5$, namely the Bloom syndrome gene product (BLM), the Werner syndrome gene product (WRN), and RECQL4, all depended on the function 
of USP28 to maintain their levels (Fig. 4E). The only exception is RECQL1 which seemed unaffected by USP 28 status. Furthermore, AZ1 treatment recapitulated the effects of USP28 depletion including the activation of CHK1 (Fig. 4F). Taken together, these results suggest that it is very likely that maintaining protein stability of RecQ family helicases underlies the requirement of USP28 in triple negative breast cancer cells.

\section{USP28 regulates the stability of RECQL5}

Having shown the requirement of USP28 for maintaining RECQL5 levels, we next sought to demonstrate that it regulates RECQL5 stability. To that end, we first determined the half-life of RECQL5 in control and USP28-depleted cells. As shown in Fig. 5A and $\mathrm{B}$, depletion of USP28 greatly reduced the half-life of RECQL5. On the other hand, overexpression of USP28 made the helicase much more stable (Fig. 5C and D). As expected, overexpression of a protease-dead version of USP28 (C171A) could not stabilize RECQL5 (Fig. 5C and D).

The results above suggest that USP28 is a deubiquitin Enzyme (DUB) for RECQL5. To demonstrate that, we first set out to determine if the two proteins interact with each other. As shown in Fig. 6A, immunoprecipitating RECQL5 could bring down USP28 and vice versa. Next, we looked at the levels of ubiquitin attached to RECQL5 to see if they were affected by USP28 status. Flag-tagged RECQL5 and shRNA against USP28 were expressed in $293 \mathrm{~T}$ cells, and the cells were then treated with and without MG132 to block proteasome activities. Immunoprecipitates obtained with anti-Flag antibodies from these cells under denaturing conditions were separated in a SDS-gel and 
blotted for detection of ubiquitin. It is clear that the depletion of USP28 resulted in much higher levels of ubiquitin associated with RECQL5, but only if the cells were treated with MG132 (Fig. 6B). Otherwise, the ubiquitinated RECQL5 was degraded.

Opposite to depleting USP28, overexpression resulted in a decrease of ubiquitination of RECQL5 (Fig. 6C), and as expected, overexpression of USP28 C171A did not cause such a decrease (Fig. 6C). Taken together, these results demonstrate that USP28 is the DUB that prevents excess ubiquitination and degradation of RECQL5 and likely other RecQ helicases as well.

\section{Other members of RecQ family helicases are also required for proliferation of TNBC} cells

Given the replication stress that triple negative breast cancer cells face and the function of RecQ helicase in DNA metabolism, it is highly likely that like RECQL5, the rest 4 helicases are also essential in TNBC cells. To determine if that is the case, we depleted BLM, WRN, RECQL1, and RECQL4 expression (Fig. S3A)with two different shRNAs in TNBC cells MDA-MB-231 (Fig. 7A), HCC1806 (Fig. S3B) and HCC1937 (Fig. S3C). All 3 TNBC cell lines ceased proliferating upon knocking down each and every RecQ family helicases.

It has been reported previously that Recql5-deficient cells are sensitive to replication stress inducers such as camptothecin [20]. We expect that USP28 depletion would also result in enhanced sensitivity to replication stress in non-TNBC cells. 
Therefore, we treated control and USP28-depleted breast cancer cells T47D with 5-FU or CPT and monitored cell viability. Indeed, USP28 knockdown cells became much more sensitive to the two chemo agents (Fig. 7B).

\section{Discussion}

Triple negative breast cancer is the more aggressive form amid all breast cancers and currently lacks effective therapies. We show here that USP28 is a potential therapeutic target of TNBC. Loss of its function prevents TNBC cells from proliferation in vitro and in vivo. Given the role of USP28 in maintaining c-MYC stability [9] and the importance of MYC in cell proliferation, we reasoned that the requirement of USP28 by TNBC cells for proliferation might be related to its function as a DUB for c-MYC. As expected, depletion of USP28 could lead to destabilization of c-MYC in TNBC cells (Fig. 3A). However, restoring c-MYC levels through overexpression could not rescue the lethality resulted from USP28 depletion (Fig. 3C), indicating that the lethality is a result of more than just a decrease in c-MYC levels (although which might still be a contributing factor).

We have shown previously that TBNC cells suffer unusually high levels of replication stress that require the function of RECQL5 to relieve [5]. Although it is unclear the source of replication stress in TNBC cells, it is likely that single strand lesions are the culprit. Interestingly, depletion of USP28 could worsen the replication stress in TNBC cells (Fig. 4A), despite the fact the DUB has been shown not to play any significant roles in DNA damage responses [13]. In addition, we did not see 
destabilization of CLASPIN upon USP28 depletion in TNBC cells but did observe enhanced activation of CHK1. These results suggest that USP28 regulates other proteins involved in DNA metabolism. Given the phenotypic similarities between USP28 depletion and that of RECQL5 in TNBC cells, we suspected that USP28 might regulate RECQL5 stability. Indeed, RECQL5 is a substrate of USP28 (Fig. 6). Depletion of USP28 resulted in more ubiquitination and destabilization of the helicase. Furthermore, we showed that not only RECQL5, but its family members are also regulated by USP28, which explains, at least in part, the failure of our attempts to rescue USP28 depletion with overexpression of RECQL5 (data not shown). Taken together, our results uncovered the link between USP 28 and the RecQ family helicases. The protection by USP 28 on these helicases seems dispensable as mice lacking Usp28 are more or less normal [12,13], but it becomes indispensable in triple negative breast cancer cells (Fig. 7C). In normal cells, although loss of USP28 does cause decreases in the levels of RecQ helicases, such decreases would presumably still leave sufficient amounts of these helicases behind to deal with the low level (if any) of replication stress. However, in the cells with induced replication stress, USP28 is no longer dispensable (Fig. 7C).

USP28 was first identified due to its function in DNA damage response through protecting CLASPIN [14]. We found here that depletion of USP28 in TNBC cells did not lead to a decrease in CLASPIN levels (Fig. 4C), suggesting that whether USP28 functions as CLASPIN's DUB is cell context dependent. Previous studies also showed that USP28 was dispensable in DNA damage response, as Usp28-deficient MEFs were not more sensitive than wildtype cells to DNA damage agents including ionizing 
radiation [13]. However, we show here that $U S P 28$ is required for cells to cope with replication stress, either arose internally such as in TNBC cells, or induced by chemotherapeutic agents (Fig. 7B).

It is interesting but not unexpected that depletion of USP28 did not lead to a reduction in c-MYC levels in MCF7 (Fig. 3A) and other non-TNBC cells (data not shown), as there are other DUBs reported to de-ubiquitinate and hence stabilize c-MYC [21-23]. Which DUB is more important or predominant for MYC stabilization is likely cell context dependent. In addition, if the E3 ubiquitin ligase(s) for MYC is not functional, there would be no need for a DUB. Of course, it also remains entirely possible that the amount of USP28 activity required to stabilize c-MYC might be cell context dependent as well. Thus, in some cells, perhaps depleting USP28 via shRNA would still leave enough DUB activity behind for c-MYC stabilization.

\section{Conclusions}

While USP28 has been shown to regulate the stability of a number of other oncogene products, such as LSD1 [10], NOTCH1 [12], etc., our data establish a new critical role played by USP28 in supporting the proliferation and viability of triple negative breast cancer cells through stabilizing RecQ family helicases and support USP28 as a therapeutic target for TNBC.

\section{Abbreviations}


TNBC: Triple-negative breast cancer; ER: Estrogen receptor; PR: Progesterone receptor; HER2: Human epidermal growth factor receptor 2; USP: Ubiquitin-specific cysteine protease; SCC: Squamous cell carcinoma; ATCC: American Type Culture Collection; DMEM: Dulbecco's modified Eagle's medium; FBS: Fetal bovine serum; UPS: Ubiquitin proteasome system; CPT: camptothecin; 5-FU: 5-Fluorouracil; MOI: Multiplicity of infection; PI: Propidium iodide; DUB: deubiquitin Enzyme; CHX: Cycloheximide; Ub: Ubiquitin.

\section{Acknowledgement}

We thank the core facilities in National Center for Protein Sciences (Beijing) and Zhejiang Provincial Key Laboratory of Pancreatic Disease for experimental support. The authors thank people in Zhang lab for helpful discussions and suggestions throughout the work.

\section{Authors' contributions}

JP and JW planned, performed experiments and analyzed data. YD, HM, LW, XZ and LT were involved in cell and animal experiments. JJ, SH and PZ participated in conceiving and designing this study. PZ drafted the manuscript. All authors read and approved the final manuscript.

\section{Funding}

This work was supported by grants from the National Natural Science Foundation of China (81773032), the National Key R\&D Program of China (2018YFA0507500). 


\section{Availability of data and materials}

All data and materials generated in this study are available from the corresponding author on reasonable request.

\section{Ethics approval and consent to participate}

Not applicable.

\section{Consent for publication}

Not applicable.

\section{Competing interests}

The authors declare no competing interest. 


\section{References}

1. de Ruijter TC, Veeck J, de Hoon JP, van Engeland M, Tjan-Heijnen VC: Characteristics of triple-negative breast cancer. Journal of cancer research and clinical oncology 2011, 137(2):183-192.

2. Reis-Filho JS, Tutt AN: Triple negative tumours: a critical review. Histopathology 2008, 52(1):108-118.

3. Lehmann BD, Bauer JA, Chen X, Sanders ME, Chakravarthy AB, Shyr Y, Pietenpol JA: Identification of human triple-negative breast cancer subtypes and preclinical models for selection of targeted therapies. The Journal of clinical investigation 2011, 121(7):2750-2767.

4. Bartkova J, Tommiska J, Oplustilova L, Aaltonen K, Tamminen A, Heikkinen T, Mistrik M, Aittomaki K, Blomqvist C, Heikkila P et al: Aberrations of the MRE11-RAD50-NBS1 DNA damage sensor complex in human breast cancer: MRE11 as a candidate familial cancer-predisposing gene. Mol Oncol 2008, 2(4):296-316.

5. Peng J, Tang L, Cai M, Chen H, Wong J, Zhang P: RECQL5 plays an essential role in maintaining genome stability and viability of triple-negative breast cancer cells. Cancer medicine 2019, 8(10):4743-4752.

6. Lakhani SR, Van De Vijver MJ, Jacquemier J, Anderson TJ, Osin PP, McGuffog L, Easton DF: The pathology of familial breast cancer: predictive value of immunohistochemical markers estrogen receptor, progesterone receptor, HER-2, and p53 in patients with mutations in BRCA1 and BRCA2. Journal of clinical oncology : official journal of the American Society of Clinical Oncology 2002, 20(9):2310-2318.

7. Turner N, Tutt A, Ashworth A: Hallmarks of 'BRCAness' in sporadic cancers. Nature reviews Cancer 2004, 4(10):814-819.

8. Shiu KK, Tan DS, Reis-Filho JS: Development of therapeutic approaches to 'triple negative' phenotype breast cancer. Expert opinion on therapeutic targets 2008, 12(9):1123-1137.

9. Popov N, Wanzel M, Madiredjo M, Zhang D, Beijersbergen R, Bernards R, Moll $\mathrm{R}$, Elledge SJ, Eilers M: The ubiquitin-specific protease USP28 is required for MYC stability. Nat Cell Biol 2007, 9(7):765-774.

10. Wu Y, Wang Y, Yang XH, Kang T, Zhao Y, Wang C, Evers BM, Zhou BP: The deubiquitinase USP28 stabilizes LSD1 and confers stem-cell-like traits to breast cancer cells. Cell reports 2013, 5(1):224-236.

11. Flugel D, Gorlach A, Kietzmann T: GSK-3beta regulates cell growth, migration, and angiogenesis via Fbw7 and USP28-dependent degradation of HIF-1alpha. Blood 2012, 119(5):1292-1301.

12. Diefenbacher ME, Popov N, Blake SM, Schulein-Volk C, Nye E, Spencer-Dene B, Jaenicke LA, Eilers M, Behrens A: The deubiquitinase USP28 controls intestinal homeostasis and promotes colorectal cancer. The Journal of clinical investigation 2014, 124(8):3407-3418.

13. Knobel PA, Belotserkovskaya R, Galanty Y, Schmidt CK, Jackson SP, Stracker TH: USP28 is recruited to sites of DNA damage by the tandem BRCT 
domains of 53BP1 but plays a minor role in double-strand break metabolism. Molecular and cellular biology 2014, 34(11):2062-2074.

14. Zhang D, Zaugg K, Mak TW, Elledge SJ: A role for the deubiquitinating enzyme USP28 in control of the DNA-damage response. Cell 2006, 126(3):529-542.

15. Prieto-Garcia C, Hartmann O, Reissland M, Braun F, Fischer T, Walz S, Schulein-Volk C, Eilers U, Ade CP, Calzado MA et al: Maintaining protein stability of Np63 via USP28 is required by squamous cancer cells. EMBO Mol Med 2020, 12(4):e11101.

16. Kok YP, Guerrero Llobet S, Schoonen PM, Everts M, Bhattacharya A, Fehrmann RSN, van den Tempel N, van Vugt M: Overexpression of Cyclin E1 or Cdc25A leads to replication stress, mitotic aberrancies, and increased sensitivity to replication checkpoint inhibitors. Oncogenesis 2020, 9(10):88.

17. Wrigley JD, Gavory G, Simpson I, Preston M, Plant H, Bradley J, Goeppert AU, Rozycka E, Davies G, Walsh $\mathrm{J}$ et al: Identification and Characterization of Dual Inhibitors of the USP25/28 Deubiquitinating Enzyme Subfamily. ACS Chem Biol 2017, 12(12):3113-3125.

18. Yada M, Hatakeyama S, Kamura T, Nishiyama M, Tsunematsu R, Imaki H, Ishida N, Okumura F, Nakayama K, Nakayama KI: Phosphorylation-dependent degradation of c-Myc is mediated by the F-box protein Fbw7. EMBO J 2004, 23(10):2116-2125.

19. Welcker M, Orian A, Jin J, Grim JE, Harper JW, Eisenman RN, Clurman BE: The Fbw7 tumor suppressor regulates glycogen synthase kinase 3 phosphorylation-dependent c-Myc protein degradation. Proc Natl Acad Sci U $S$ A 2004, 101(24):9085-9090.

20. $\mathrm{Hu} \mathrm{Y,} \mathrm{Lu} \mathrm{X,} \mathrm{Zhou} \mathrm{G,} \mathrm{Barnes} \mathrm{EL,} \mathrm{Luo} \mathrm{G:} \mathrm{Recql5} \mathrm{plays} \mathrm{an} \mathrm{important} \mathrm{role} \mathrm{in}$ DNA replication and cell survival after camptothecin treatment. Mol Biol Cell 2009, 20(1):114-123.

21. Sobol A, Askonas C, Alani S, Weber MJ, Ananthanarayanan V, Osipo C, Bocchetta M: Deubiquitinase OTUD6B Isoforms Are Important Regulators of Growth and Proliferation. Mol Cancer Res 2017, 15(2):117-127.

22. Kim D, Hong A, Park HI, Shin WH, Yoo L, Jeon SJ, Chung KC: Deubiquitinating enzyme USP22 positively regulates c-Myc stability and tumorigenic activity in mammalian and breast cancer cells. $J$ Cell Physiol 2017, 232(12):3664-3676.

23. Pan J, Deng Q, Jiang C, Wang X, Niu T, Li H, Chen T, Jin J, Pan W, Cai X et al: USP37 directly deubiquitinates and stabilizes c-Myc in lung cancer. Oncogene 2015, 34(30):3957-3967. 


\section{Figure Legend}

\section{Figure 1. USP28 sustains the proliferation of TNBC cells.}

(A) Growth Analysis of MDA-MB-231, HCC1806, and HCC1937 cells depleted of USP28 expression via two independent shRNAs targeting the 3'-UTR region.

(B) Immunoblot analysis of USP28 levels in SUM159 cells with USP28 overexpression first and then depleted of endogenous USP28 expression via the same shRNAs in (A).

(C) Colony formation assay of the SUM159 cells from (B).

(D) Representative images and (E) quantification of HCC1806 cells treated with $0,10,15$ or $20 \mu \mathrm{M}$ AZ1 for 48 hrs.

(F) Cell viability assay of MDA-MB-231 and HCC1937 upon treatment with 0, 10, 15, 20,25 or $30 \mu \mathrm{M}$ AZ1 for $48 \mathrm{hrs}$.

\section{Figure 2. Targeting USP28 impedes the growth of HCC1806 xenografts.}

(A) HCC1806 cells infected with shNC or shUSP28 lentiviruses were innoculated subcutaneously into female Balb/c nude mice. The tumors were collected three weeks after the inoculation. Tumor weight and size were measured. Data are mean \pm SD $(n=5$ per group). Student's t test: *, p<0.05; **, p<0.01; ***, p<0.001. (B) HCC1806 cells were innoculated subcutaneously into female Balb/c nude mice. When the average tumor size reached $100 \mathrm{~mm}^{3}$, the mice were divided randomly into two groups ( $n=7$ per group). One group were treated with vehicle, another with AZ1 (200 $\mathrm{mg} / \mathrm{kg}$, oral gavage) every day. The tumor sizes were measured every three days and the tumors were harvested 12 days after the treatment. Data are mean $\pm \mathrm{SD}$. Student's t test: $*, p<0.05$. 
Figure 3. USP28 depletion destabilizes c-MYC and induces cell cycle arrest in TNBC cells.

(A) Immunoblot analysis of c-MYC in MDA-MB-231, HCC1937, HCC1806 and MCF7 cells infected with lentiviruses expressing control or USP28 shRNAs.

(B) Cell cycle distribution in MDA-MB-231, HCC1937 and MCF7 cells with or without USP28 depletion. The percentage of the cells in each cell cycle phase were calculated with ModFit LT software.

(C) Overexpression of c-MYC-T58A fails to rescue the growth arrest imposed by USP28 depletion MDA-MB-231 cells. The expression of c-MYC (endogenous and exogenous T58A mutant) as well as USP28 was analyzed with immunoblotting and the cells were subjected to growth curve analysis.

Figure 4. USP28 inhibition induces accumulation of DNA damage and activation of DNA damage checkpoint in TNBC cells.

(A). Immunofluorescence staining and quantification of DNA damage marker $\gamma \mathrm{H} 2 \mathrm{AX}$ focus in USP28-depleted MDA-MB-231 cells. Student's t test: ***, p<0.001.

(B). Immunoblot analysis of CHK1 in MDA-MB-231 cells depleted of USP28.

(C). Immunoblot analysis of CLASPIN in MDA-MB-231 cells depleted of USP28.

(D). Immunoblot analysis of RECQL5 in MDA-MB-231, SUM159 and T47D cells depleted of USP28.

(E). Immunoblot analysis of RecQ family helicases in MDA-MB-231 cells depleted of USP28. 
(F). Immunoblot analysis of relative proteins in HCC1806 cells treated with USP28 inhibitor AZ1 for 48 hrs.

\section{Figure 5. USP28 stabilizes RECQL5.}

(A). Analysis of RECQL5 stability in a cycloheximide chase experiment. 293T cells were transfected with Flag-RECQL5 first and then depleted of USP28 expression, treated with $100 \mu \mathrm{g} / \mathrm{ml}$ cycloheximide $(\mathrm{CHX})$, and harvested at different time points for analysis.

(B). Quantification of Flag-RECQL5 levels in (A).

(C). Overexpression of USP28 stabilizes RECQL5. 293T cells with USP28 or USP $28^{C 171 A}$ overexpression were subjected to the same CHX chase experiment as in (A) except the endogenous RECQL5 was examined.

(D). Quantification of RECQL5 levels in (C).

\section{Figure 6. USP28 is a DUB for RECQL5.}

(A). USP28 interacts RECQL5. 293T cells were transfected with Flag-RECQL5 and cell lysates were prepared. Flag-RECQL5 was immunoprecipitated with M2 beads and USP28 with anti-USP28 antibodies from the cell lysates. The precipitates were then subjected to immunoblotting analysis.

(B). Depleting USP28 expression enhances RECQL5 ubiquitination. 293T cells with ectopic expression of Flag-RECQL5 were transduced with shUSP28 or control shRNA, treated with $10 \mu \mathrm{M}$ MG132 for 8 hours, and immunoprecipitated with M2 beads. The precipitates were then subjected to immunoblotting analysis with antibodies against ubiquitin, Flag, and USP28. 
(C). Overexpression of USP28 reduces RECQL5 ubiquitination. 293T cells were transfected with Flag-RECQL5, HA-ubiquitin (HA-Ub), and USP28 WT or USP28

C171A, treated with $10 \mu \mathrm{M}$ MG132 for 8 hours, and immunoprecipitated with M2 beads. The precipitates were then subjected to immunoblotting analysis with antibodies against HA, Flag, and USP28.

Figure 7. RecQ family helicases are required for the proliferation of TNBC cells. (A). The growth curve analysis of MDA-MB-231 cells depleted of RecQ helicases. Each helicase was knocked down via two independent shRNAs.

(B). Depletion of RECQL5 or USP28 sensitized T47D cells to replication stress inducers 5-FU and camptothecin.

(C). A schematic model illustrating the function of USP28 in helping replication stress relief in TNBC cells through RecQ family helicases, thereby maintaining the viability of TNBC cells. 
A

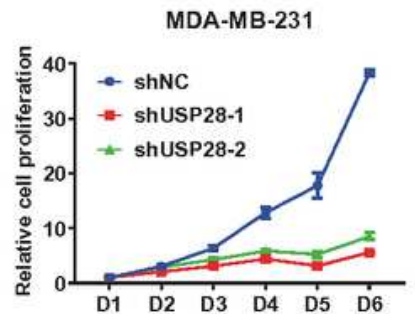

B

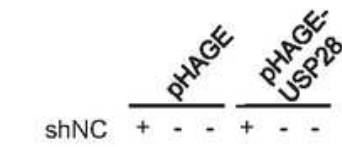

ShUSP28-1 - + . + -

shUSP28-2 - - + - - +

AN-USP28

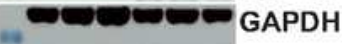

D

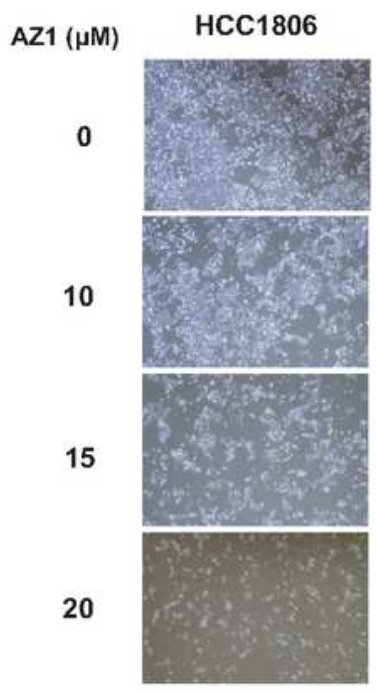

C
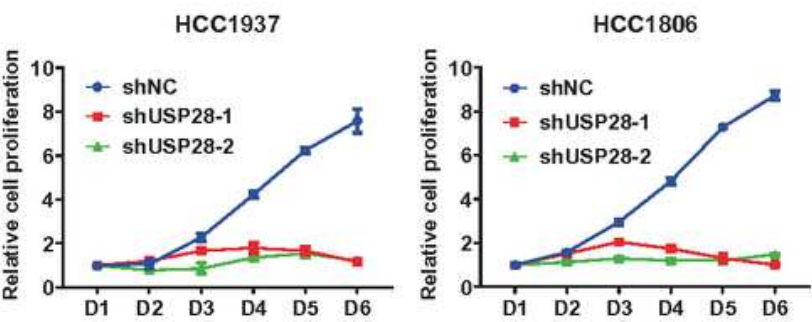

E

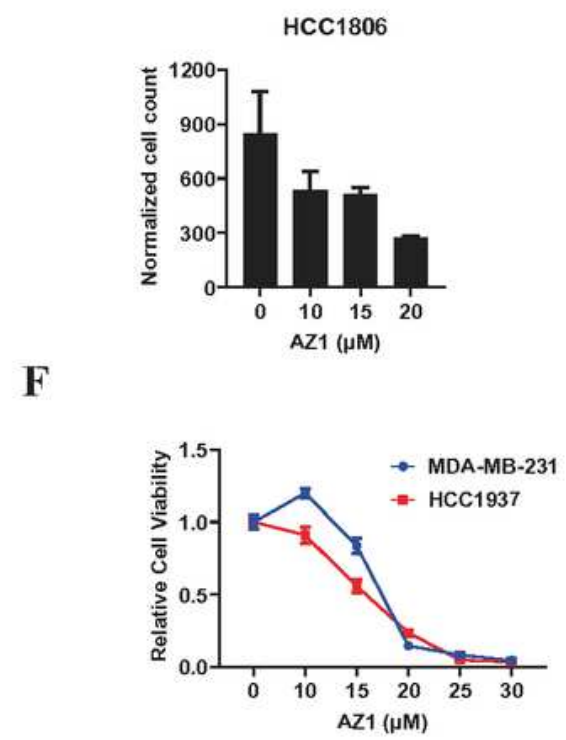

F

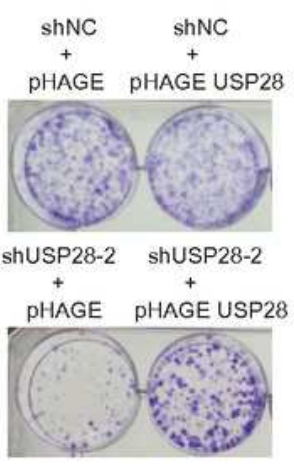

shUSP28-1 shUSP28-1

pHAGE PHAGEUSP28

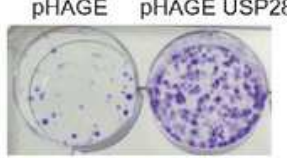

Figure 1

USP28 sustains the proliferation of TNBC cells. (A) Growth Analysis of MDA MB 231, HCC1806, and HCC1937 cell s depleted of USP28 expression via two independent shRNAs targeting the 3' UTR region. (B) Immunoblot analysis of USP28 levels i n SUM159 cells with USP28 overexpression first and then 
depleted of endogenous USP28 expression via the same shRNAs in (A). (C) Colony formation assay of the SUM159 cell s from (B). (D) Representative images and (E) quantification of HCC1806 cells treated with $0,10,15$ or $20 \mu \mathrm{M}$ AZ1 for $48 \mathrm{hrs}$. (F) Cell viability assay of MDA-MB-231 and HCC1937 upon treatment with $0,10,15,20,25$ or $30 \mu \mathrm{M}$ AZ1 for 48 hrs.

A
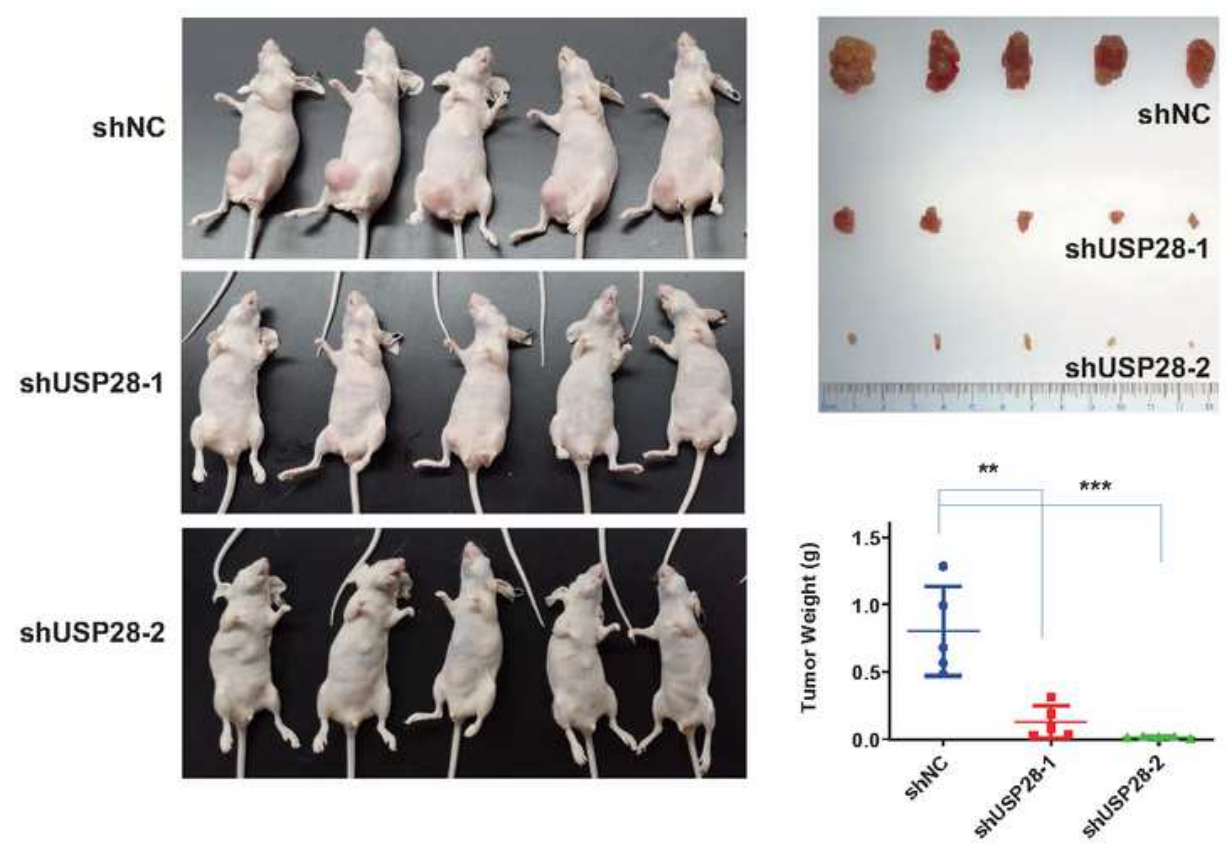

B

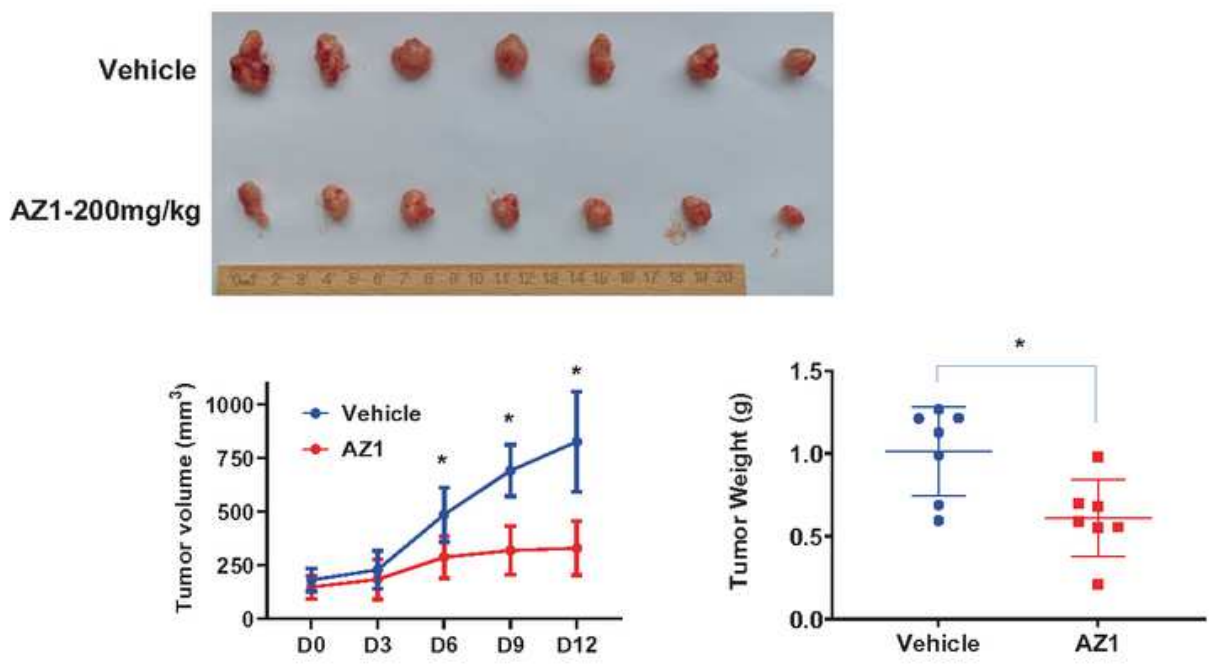

Figure 2 
Targeting USP28 impedes the growth of HCC1806 xenografts. (A) HCC1806 cells infected with shNC or shUSP28 lentiviruses were innoculated subcutaneously into female Balb/c nude mice. The tumors were collected three weeks after the inoculation. Tumor weight and size were measured. Data are mean \pm SD ( $n=5$ per group). Student's t test: ${ }^{*}, p<0.05 ; * \star, p<0.01 ; * * *, p<0.001$. (B) HCC1806 cells were innoculated subcutaneously into female Balb/c nude mice. When the average tumor size reached $100 \mathrm{~mm}$, the mice were divided randomly into two groups ( $n=7$ per group). One group were treated with vehicle, another with AZ1 (200 mg/kg, oral gavage) every day. The tumor sizes were measured every three days and the tumors were harvested 12 days after the treatment. Data are mean \pm SD. Student's $t$ test: *, $p<0.05$. 
A

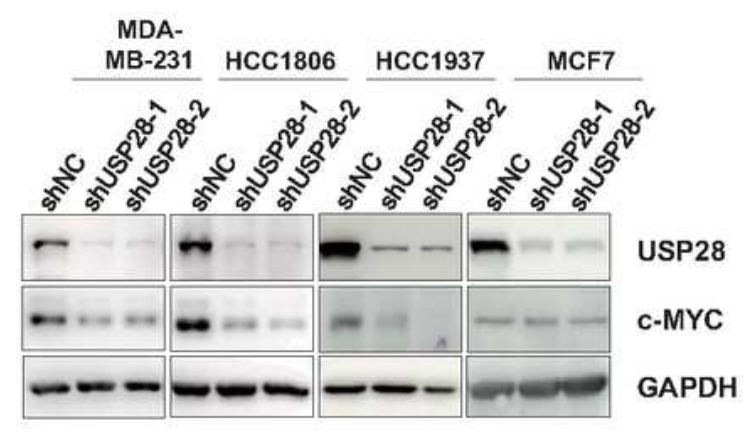

B
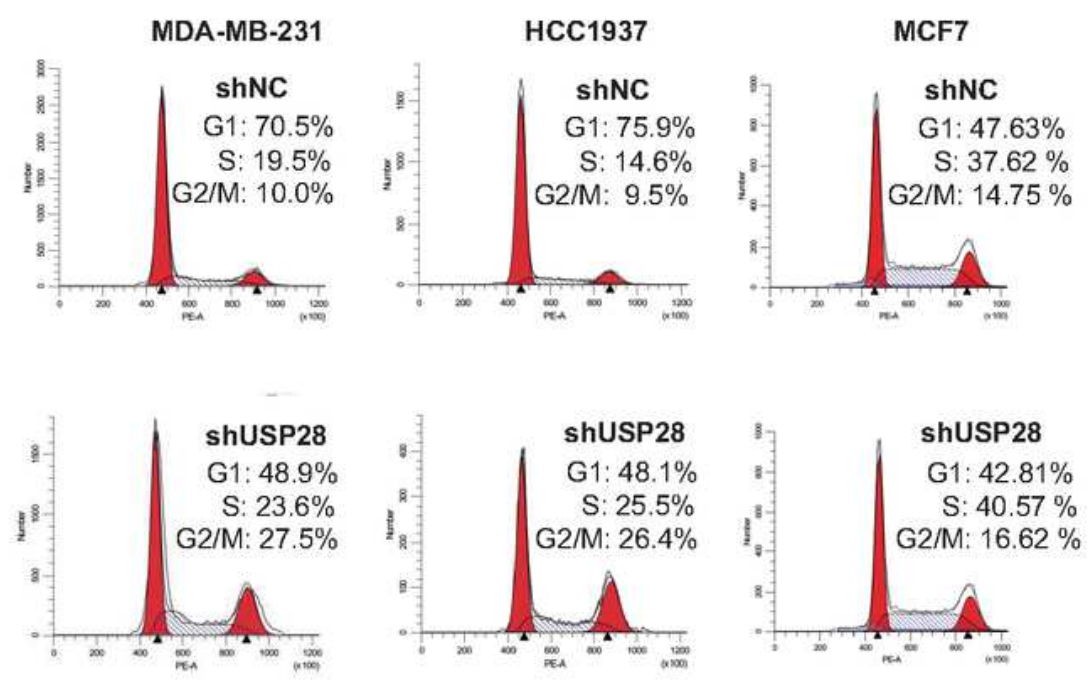

C
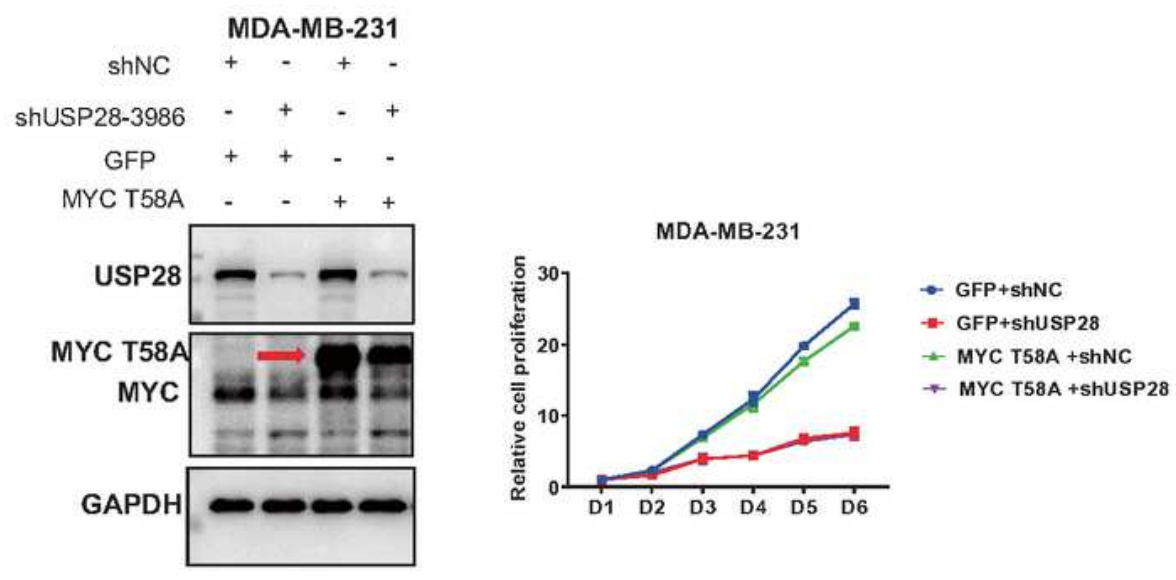

\section{Figure 3}

USP28 depletion destabilizes c-MYC and induces cell cycle arrest in TNBC cells. (A) Immunoblot analysis of c-MYC in MDA-MB-231, HCC1937, HCC1806 and MCF7 cells infected with lentiviruses expressing control or USP28 shRNAs. (B) Cell cycle distribution in MDA-MB-231, HCC1937 and MCF7 cells with or without USP28 depletion. The percentage of the cells in each cell cycle phase were calculated with ModFit LT software. (C) Overexpression of C-MYC-T58A fails to rescue the growth arrest imposed by 
USP28 depletion MDA-MB-231 cells. The expression of c-MYC (endogenous and exogenous T58A mutant) as well as USP28 was analyzed with immunoblotting and the cells were subjected to growth curve analysis.

A

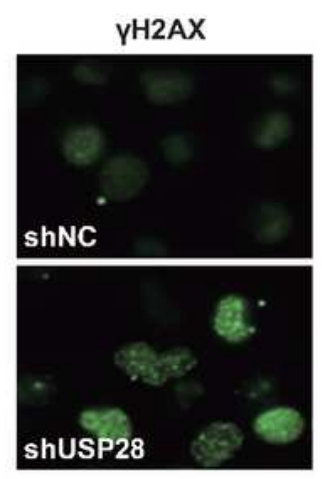

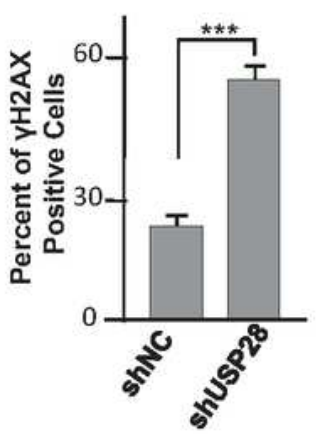

B

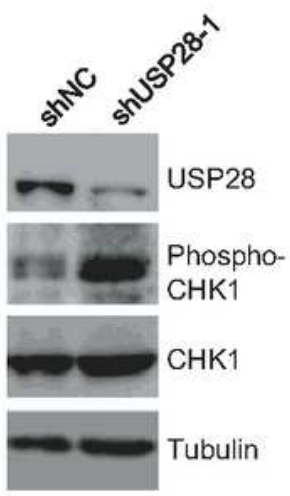

C

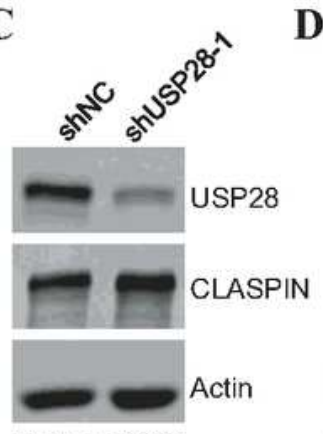

MDA-MB-231
D

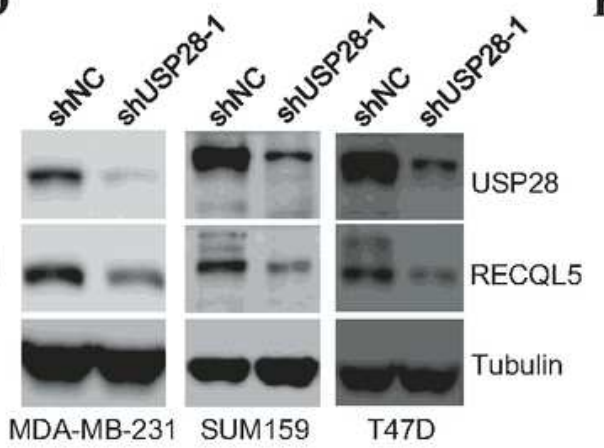

F

Figure 4

USP28 inhibition induces accumulation of DNA damage and activation of DNA damage checkpoint in TNBC cells. (A). Immunofluorescence staining and quantification of DNA damage marker $\mathrm{YH} 2 \mathrm{AX}$ focus in 
USP28-depleted MDA-MB-231 cells. Student's t test: ${ }^{\star \star \star}, p<0.001$. (B). Immunoblot analysis of CHK1 in MDA-MB-231 cells depleted of USP28. (C). Immunoblot analysis of CLASPIN in MDA-MB-231 cells depleted of USP28. (D). Immunoblot analysis of RECQL5 in MDA-MB-231, SUM159 and T47D cells depleted of USP28. (E). Immunoblot analysis of RecQ family helicases in MDA-MB-231 cells depleted of USP28. (F). Immunoblot analysis of relative proteins in HCC1806 cells treated with USP28 inhibitor AZ1 for 48 hrs.

A

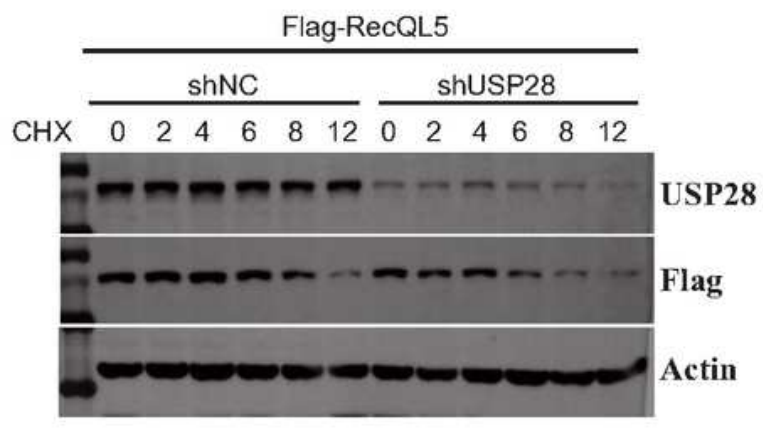

B

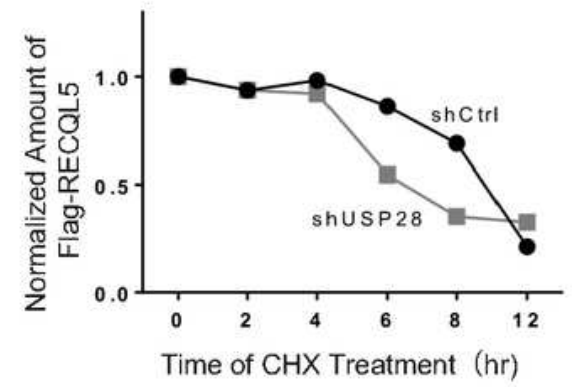

C

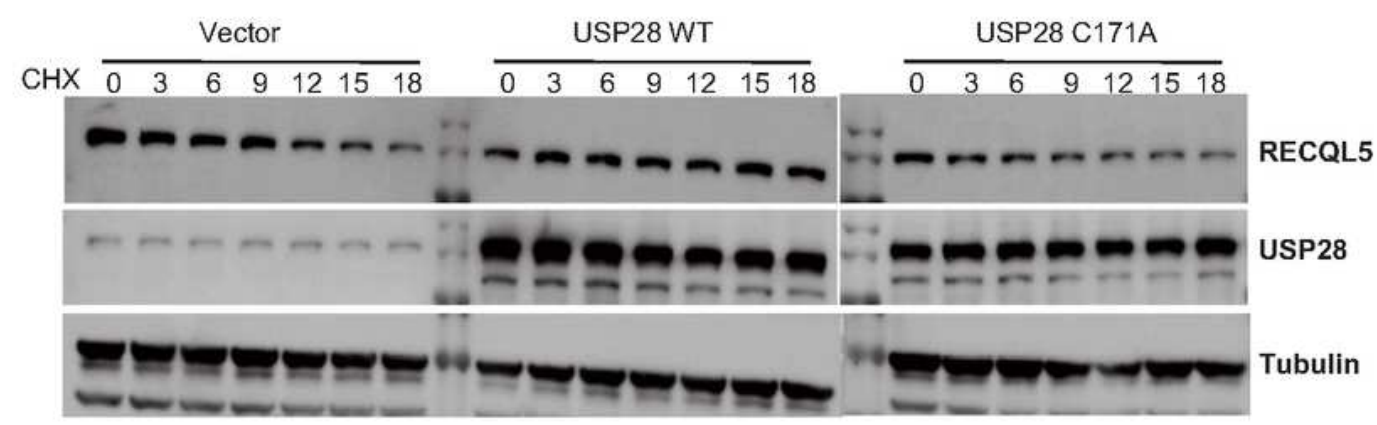

D

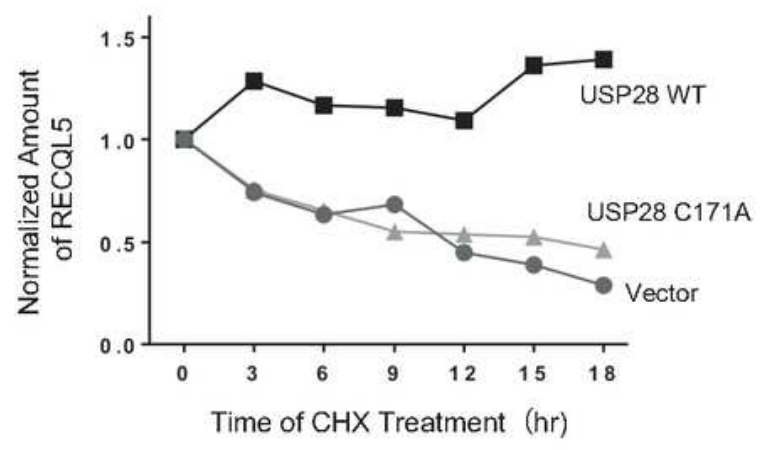

Figure 5 
USP28 stabilizes RECQL5. (A). Analysis of RECQL5 stability in a cycloheximide chase experiment. 293T cells were transfected with Flag-RECQL5 first and then depleted of USP28 expression, treated with $100 \mu \mathrm{g} / \mathrm{ml}$ cycloheximide (CHX), and harvested at different time points for analysis. (B). Quantification of Flag-RECQL5 levels in (A). (C). Overexpression of USP28 stabilizes RECQL5. 293T cells with USP28 or USP28C171A overexpression were subjected to the same $\mathrm{CHX}$ chase experiment as in (A) except the endogenous RECQL5 was examined. (D). Quantification of RECQL5 levels in (C). Figure 6.

A

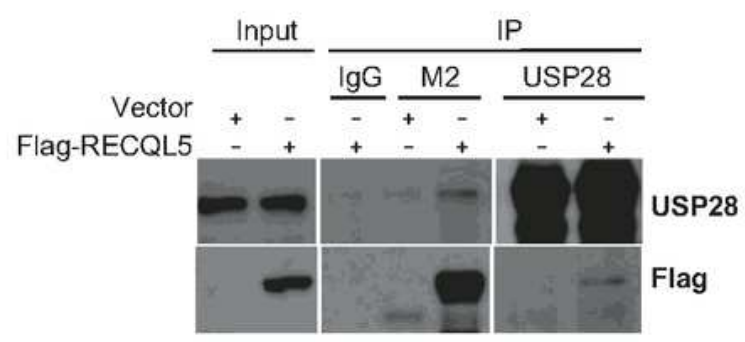

B

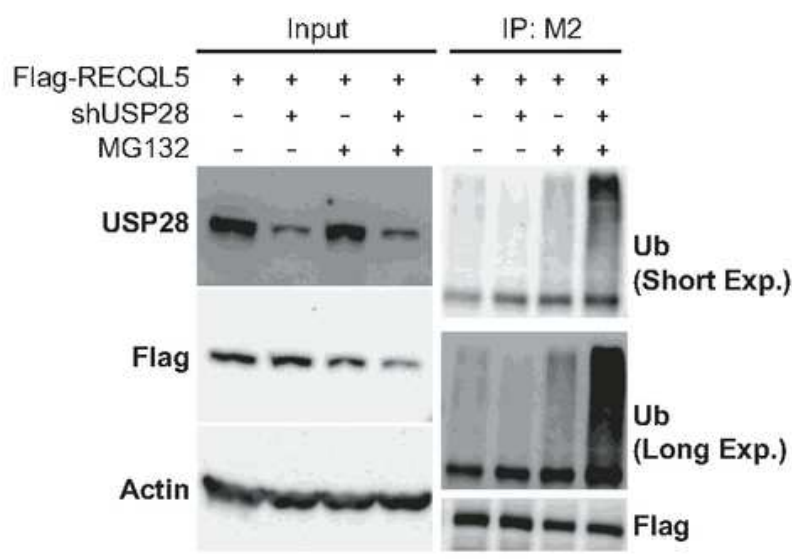

C

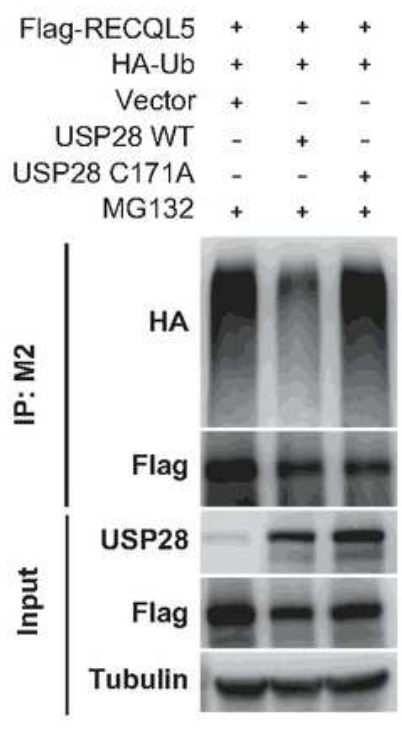


USP28 is a DUB for RECQL5. (A). USP28 interacts RECQL5. 293T cells were transfected with FlagRECQL5 and cell lysates were prepared. Flag-RECQL 5 was immunoprecipitated with M2 beads and USP28 with anti-USP28 antibodies from the cell lysates. The precipitates were then subjected to immunoblotting analysis. (B). Depleting USP28 expression enhances RECQL5 ubiquitination. 293T cells with ectopic expression of Flag-RECQL5 were transduced with shUSP28 or control shRNA, treated with $10 \mu \mathrm{M}$ MG132 for 8 hours, and immunoprecipitated with M2 beads. The precipitates were then subjected to immunoblotting analysis with antibodies against ubiquitin, Flag, and USP28. 28 (C). Overexpression of USP28 reduces RECQL5 ubiquitination. 293T cells were transfected with Flag-RECQL5, HA-ubiquitin (HA-

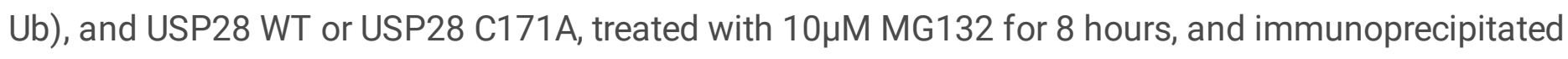
with $\mathrm{M} 2$ beads. The precipitates were then subjected to immunoblotting analysis with antibodies against HA, Flag, and USP28. 
A
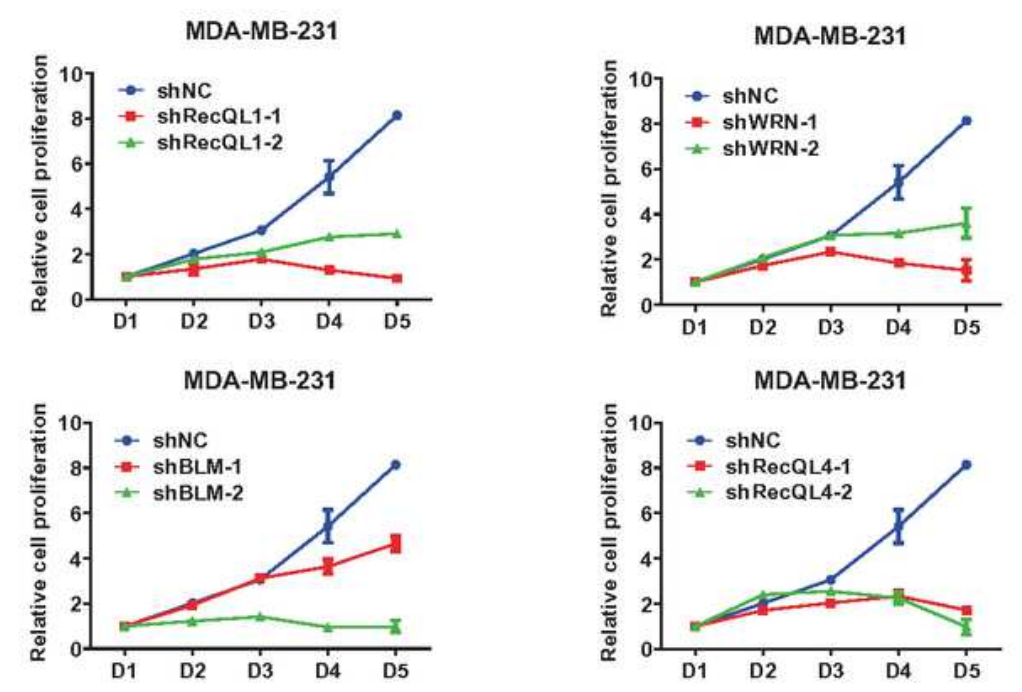

$\mathbf{B}$

T47D

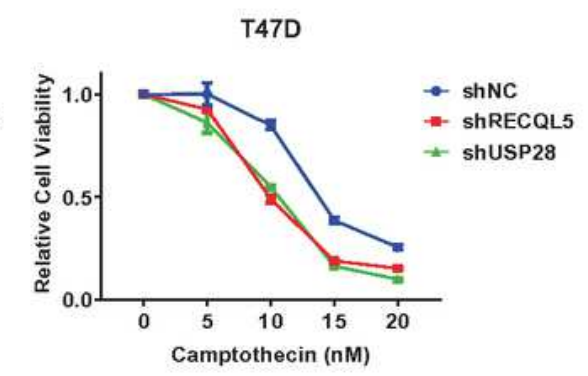

C
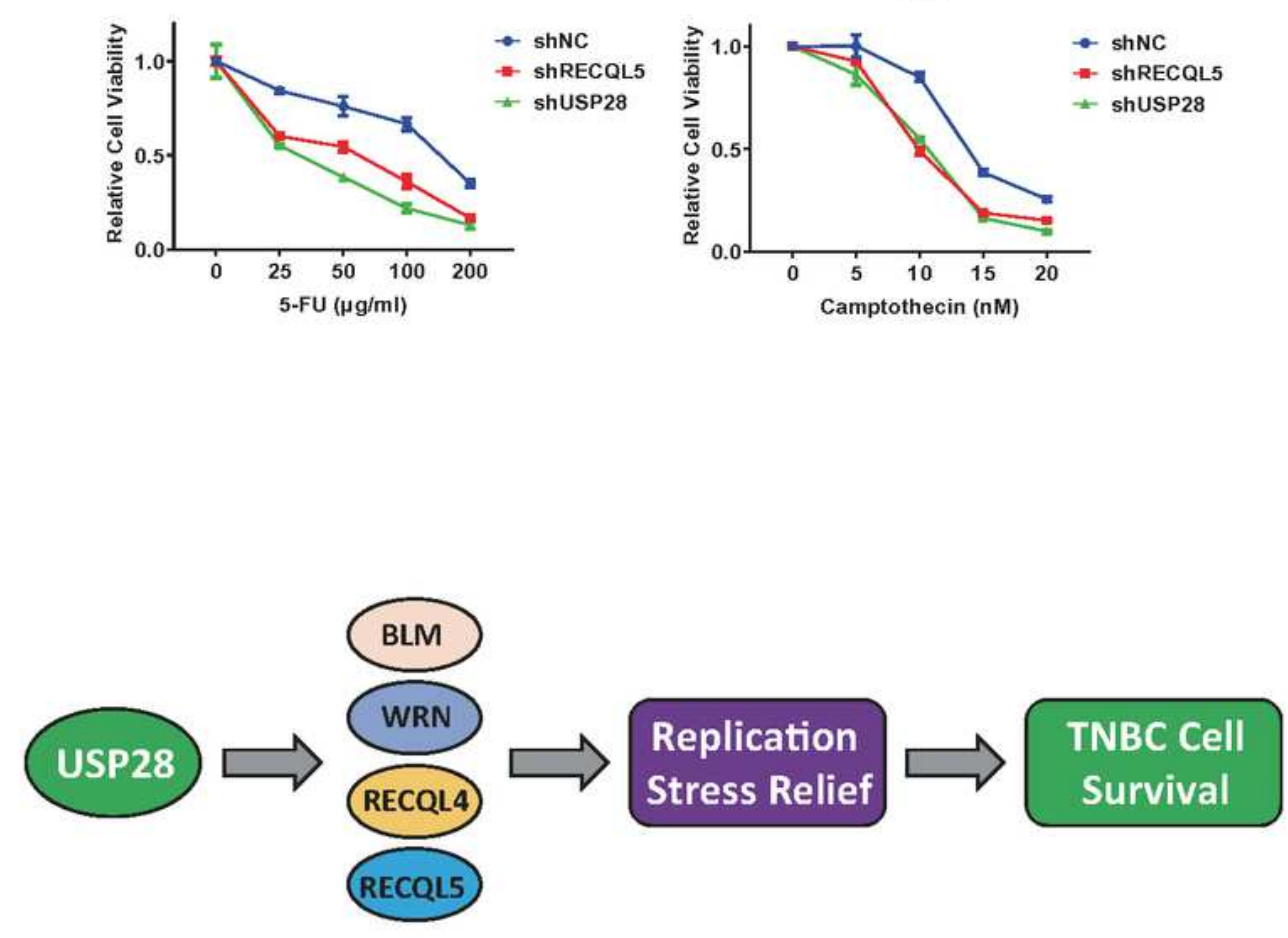

\section{Figure 7}

RecQ family helicases are required for the proliferation of TNBC cells. (A). The growth curve analysis of MDA-MB-231 cells depleted of RecQ helicases. Each helicase was knocked down via two independent shRNAs. (B). Depletion of RECQL5 or USP28 sensitized T47D cells to replication stress inducers 5-FU and camptothecin. (C). A schematic model illustrating the function of USP28 in helping replication stress relief in TNBC cells through RecQ family helicases, thereby maintaining the viability of TNBC cells. 


\section{Supplementary Files}

This is a list of supplementary files associated with this preprint. Click to download.

- Supplementarymaterial.pdf 